\title{
Proprieties of adhesive surface arrays to thyroid cartilage for recurrent laryngeal nerve monitoring
}

\author{
Yishen Zhao ${ }^{1}$, Daqi Zhang ${ }^{1}$, Le Zhou ${ }^{1}$, Shijie Li $^{1}$, Tie Wang ${ }^{1}$, Fang Li $^{1}$, Yujia Han ${ }^{1}$, Gianlorenzo Dionigi ${ }^{2}$, \\ Hui Sun ${ }^{1}$ \\ ${ }^{1}$ Division of Thyroid Surgery, China-Japan Union Hospital of Jilin University, Jilin Provincial Key Laboratory of Surgical Translational Medicine, \\ Jilin Provincial Precision Medicine Laboratory of Molecular Biology and Translational Medicine on Differentiated Thyroid Carcinoma, Changchun, \\ China; ${ }^{2}$ Division for Endocrine and Minimally Invasive Surgery, Department of Human Pathology in Adulthood and Childhood “Gaetano Barresi”, \\ University Hospital G. Martino, University of Messina, Messina, Italy \\ Contributions: (I) Conception and design: All Authors; (II) Administrative support: H Sun; (III) Provision of study materials or patients: H Sun; (IV) \\ Collection and assembly of data: Y Zhao; (V) Data analysis and interpretation: Y Zhao; (VI) Manuscript writing: All authors; (VII) Final approval of \\ manuscript: All authors. \\ Correspondence to: Hui Sun. Division of Thyroid Surgery, China-Japan Union Hospital of Jilin University, Jilin Provincial Key Laboratory of Surgical \\ Translational Medicine, Jilin Provincial Precision Medicine Laboratory of Molecular Biology and Translational Medicine on Differentiated Thyroid \\ Carcinoma, Changchun, China. Email: s_h@jlu.edu.cn.
}

Background: To investigate the optimal placement and size of adhesive thyroid cartilage electrodes (ATCEs) placed on the thyroid cartilage in porcine models for evaluating recurrent laryngeal nerve function compared with the other 3 recording electrode types.

Methods: Four Meishan piglets were used for this study. The electromyogram (EMG) signal stability was detected by intraoperative nerve monitoring (IONM). Best location and size of ATCEs were detected, and the EMG signals from each were compared. Latency data were tested by stimulating the different nerve points, and 6 manipulations of the trachea and endotracheal tube (ET) were applied to test the stability of EMG signals. ET electrodes, needle electrodes, and transcutaneous recording electrodes were simultaneously tested with comparison to ATCEs.

Results: The optimal placement locations and sizes of ATCEs were determined. The amplitudes and latencies recorded from the ATCEs were consistent with those of the ET electrodes. More anti-interference was observed with the ATCEs than with the ET electrodes in surgical manipulations. ATCEs could be used during intermittent and continuous monitoring in similar fashion to ET electrodes and needle electrodes.

Conclusions: ATCEs had consistent monitoring function with ET electrodes and needle electrodes. Feasibility, EMG stability, and optimal location and size of ATCEs for IONM were resolved. The EMG profiles from the ATCEs were more stable during surgical manipulations.

Keywords: Intraoperative neuromonitoring; recording electrodes; adhesive electrodes; thyroid surgery; porcine model

Submitted Feb 23, 2021. Accepted for publication Apr 17, 2021.

doi: $10.21037 / \mathrm{atm}-21-1229$

View this article at: http://dx.doi.org/10.21037/atm-21-1229

\section{Introduction}

Since intraoperative neuromonitoring (IONM) has been recognized as the leading technology of thyroid and parathyroid surgery, medical electrodes enabling the measurement of recurrent laryngeal nerve (RLN) signals, such as in electromyography (EMG), have been extensively researched (1-5). Integrating medical electrodes into the endotracheal tube (ET) in particular makes it possible to remotely monitor patients' laryngeal nerves, thereby providing essential information as a means to diagnose 
impending RLN stress in the early stages (6-8).

ET electrodes record the EMG signal of laryngeal muscles, mainly the thyroarytenoid muscles, by insertion directly against the mucosa overlying the intrinsic true vocal cords (8-10). The disadvantages of ETs include expense and vulnerability to manipulation, the collection of saliva interfering with the acquisition of optimal signals $(6,7)$, and the inability to use electrodes in patients with tracheostomy or those with giant goiters compressing the trachea (11-13). Above all, the primary disadvantage is that changes in the position of the ET electrode can affect the EMG potentials during monitored thyroid surgery. To avoid low EMG amplitudes or monitoring failure, the tube size should be chosen for optimal contact with the vocal cords 6 , and the location of the ET electrodes in contact with the vocal cords should be fairly accurate. Displacement of the ET in any direction can cause an unstable or even false loss of EMG signal, yet positioning is complicated and difficult without a visual laryngoscope, and the difficulty is compounded after the tube has been fixed during the surgery $(7-10)$. Finally, the position of the trachea must be fixed, as any intraoperative alteration can affect the recording function of the ET electrodes (13).

Other types of recording electrodes have been proven to be useful for monitoring the RLN during thyroid surgery; these include thyroid cartilage (TC) needle electrodes and transcutaneous recording electrodes (7,13-15). A previous study showed that TC needle electrodes measured higher amplitudes and more stable EMG signals than that from ET electrodes, and the EMG recording parameters were not affected by manipulation during surgery (13). Nonetheless, TC needle recording electrodes are invasive, with associated risks such as insertion trauma, vocal cord or laryngeal hematoma, vocal cord infection, ET cuff deflation, and the retention of fractured needle segments $(6,7,13)$. Furthermore, the procedure requires extra care in elderly patients with calcified TC $(7,16)$. The method of transcutaneous recording electrodes seems to have addressed these issues, but this approach is unable to record effective amplitudes and can easily be affected by surgical manipulations on the skin (15).

In this study, we developed biocompatible, electrodes that self-adhere to TC for RLN EMG measurements. We used a porcine model to investigate the feasibility and stability of adhesive TC electrodes (ATCEs) for monitoring the RLN. The quality of ATCE signals were evaluated at various locations on the TC surface and with varying sizes of ATCEs. In addition, the monitoring functions of ET,
TC needle, and transcutaneous recording electrodes, were compared.

We present the following article in accordance with the ARRIVE reporting checklist (available at http://dx.doi. org/10.21037/atm-21-1229).

\section{Methods}

\section{Compliance with ethical standards}

Experiments were performed under a project license (No.: 2019072801) granted by ethics board of Jilin Provincial Key Laboratory of Surgical Translational Medicine, in compliance with national or institutional guidelines for the care and use of animals.

\section{Materials}

Four Meishan female piglets were purchased from Medtronic Innovation Center (MIC, Shanghai, China), aged $1.4 \pm 0.4$ months with an average weight of $23.8 \pm 1.8 \mathrm{~kg}$, and a range of 22.4 to $25.2 \mathrm{~kg}$. The piglets were provided adequate veterinary care and were fasted for 6 hours but allowed water before the experiment. The Institutional Animal Care and Use Program Committee for Research of Jilin University approved this prospective experimental study. The research was conducted in accordance with guidelines of Animal Research Reporting of In Vivo Experiments (ARRIVE) $(17,18)$. All experiments on living animals were exclusively performed by or under the supervision of attending veterinarian personnel. All procedures were performed by Surgeons of the Division of Thyroid Surgery, China-Japan Union Hospital of Jilin University.

\section{Preoperative preparation}

The piglets received induction anesthesia with atropine sulphate $(0.5 \mathrm{mg})$ via subcutaneous injection, and tiletamine/ zolazepam (zoletil $40 \mathrm{mg}, 2 \mathrm{mg} / \mathrm{kg}$ ) and xylazine hydrochloride (40 mg, $2 \mathrm{mg} / \mathrm{kg}$ ) intramuscularly. EMG electrode surface ETs (standard reinforced 7.0\# internal diam eter, Medtronic, Jacksonville, FL, USA) were properly inserted by an anesthesiologist (6). The depth and angle of the contact between the electrode surface of the ET and the mucosa of the vocal cord was pre- and intraoperatively confirmed by video laryngoscopy (6). Muscle relaxants were avoided during all procedures (6). General anesthesia was maintained using isoflurane $(2.0-3.0 \%)$ and oxygen $(2.0 \mathrm{~L} / \mathrm{min})(6)$. 

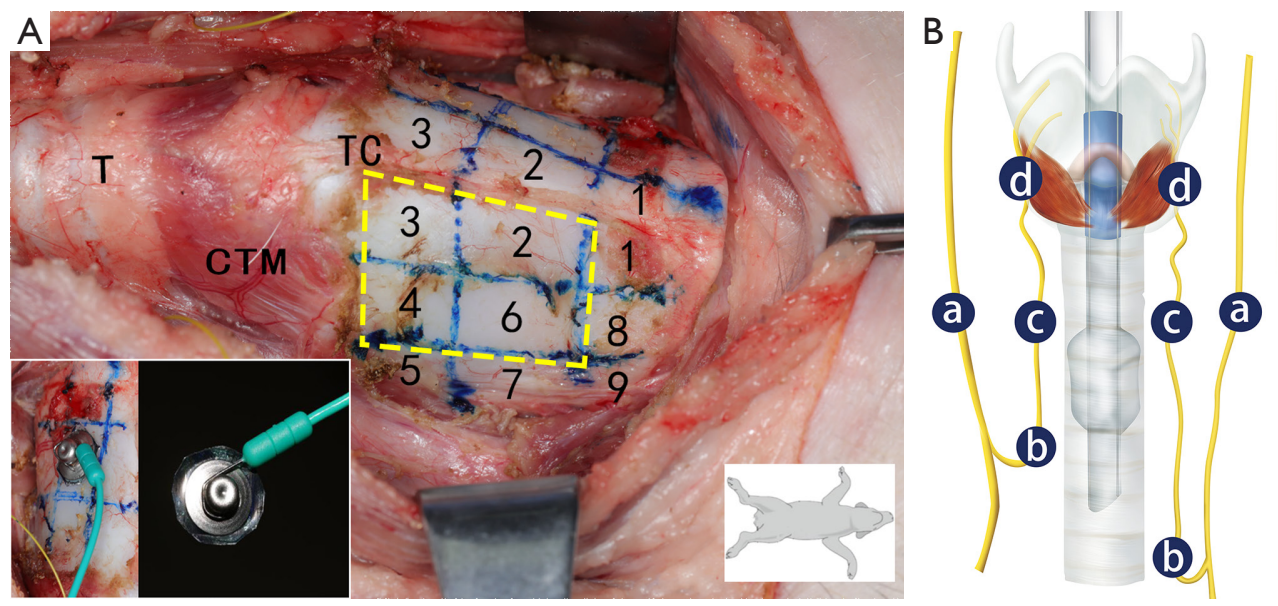

Figure 1 The method of thyroid cartilage subarea, fixing and the stimulating points on laryngeal nerves. (A) The TC is described by length and width on each side. Each side of the TC was divided into 9 areas with 2 transverse and 2 sagittal lines. The superior horizontal line divided the anterior TC horn into 2 parts: superior and inferior. The inferior horizontal line was at the middle section of the inferior part of the anterior TC horn, and parallel to the upper horizontal line. Two vertical lines were parallel to the anterior horn of the TC, the medial line began with the inner part of the rectus of the CTM, and the lateral line began with the inner part of the obliquus of the CTM. Each area was about $1 \mathrm{~cm}^{2}$. Each area was numbered (No. 1-9). Areas 1, 5, and 7-9 were removed because of anatomic difficulties, for placement of any size of the ATCEs. Therefore, only areas 2-4, and 6 (yellow dashed box) were evaluated in this study. (B) Left, ATCE during surgery. Right, a detailed image of an ATCE with a diameter of $13 \mathrm{~mm}$. (C) Schematic illustration of the 4 locations of stimulation: (a) the vagus nerve located at the distal end of the APS electrode; (b) the proximal end of the RLN; (c) the middle section of the RLN; (d) the RLN at the laryngeal entry point. TC, thyroid cartilage; ATCEs, adhesive thyroid cartilage electrodes; CTM, cricothyroid muscle; T, trachea; RLN, recurrent laryngeal nerve; APS, Automatic Periodic Stimulation.

\section{IONM facilities and equipment}

The electrically evoked EMG of the thyroarytenoid muscles and lateral cricoarytenoid muscles was simultaneously registered by ATCE (experiment) and ET surface electrodes (control) in 4 piglets. Nerve monitoring system software (NIM 3.0, Medtronic, Jacksonville, FL, USA) was applied to register the EMG. The event threshold was set at $100 \mu \mathrm{V}$ (6). Single-use, incrementing Prass monopolar standard flexible tip stimulating probes (No. 8225490; Medtronic, Jacksonville, FL, USA) were used for nerve stimulations, with a 100-ms impulse duration and a 4-Hz frequency.

\section{EMG profile measurement}

The following EMG potentials were recorded: amplitude, latency, and threshold. To more precisely and efficiently observe changes in the EMG in real time, we used C-IONM with $2.0 \mathrm{~mm}$ Automatic Periodic Stimulation (APS; Medtronic, Jacksonville, FL, USA), which is a monopolar cuff electrode that is positioned on the vagus nerve (15). After connecting the APS electrode with the NIM 3.0 system, baselines for the latency and amplitude of the evoked response were calibrated automatically to serve as control data. The upper limit of latency $(+10 \%)(6)$ and the lower limit of the amplitude (-50\%) (15) were set as alarm lines. Signal failure implied absence of signal as did an $\mathrm{EMG}$ signal $<100 \mu \mathrm{V}$ from a primary intact signal with adequate stimulation of 1 to $2 \mathrm{~mA}$ (6).

\section{ATCE material fabrication and characterization}

Sets of commercially available ATCEs with different dimensions were used and trimmed with a single needle electrode (Medtronic Xomed, Jacksonville, FL, USA) and electrocardiography (ECG) conductive adhesive hydrogel (Covidien, Mansfield, MA, USA). The body of the needle electrode was curved and hooked at the metal protrusion of the ECG conductive adhesive hydrogel, thus forming a conductive path (Figures 1,2) (18-21). The trimmed ATCEs were self-adhesive, and could stick to the TC surface without the use of any gel or adhesive. During the surgery, 2 ATCEs were pasted symmetrically on both sides of the 

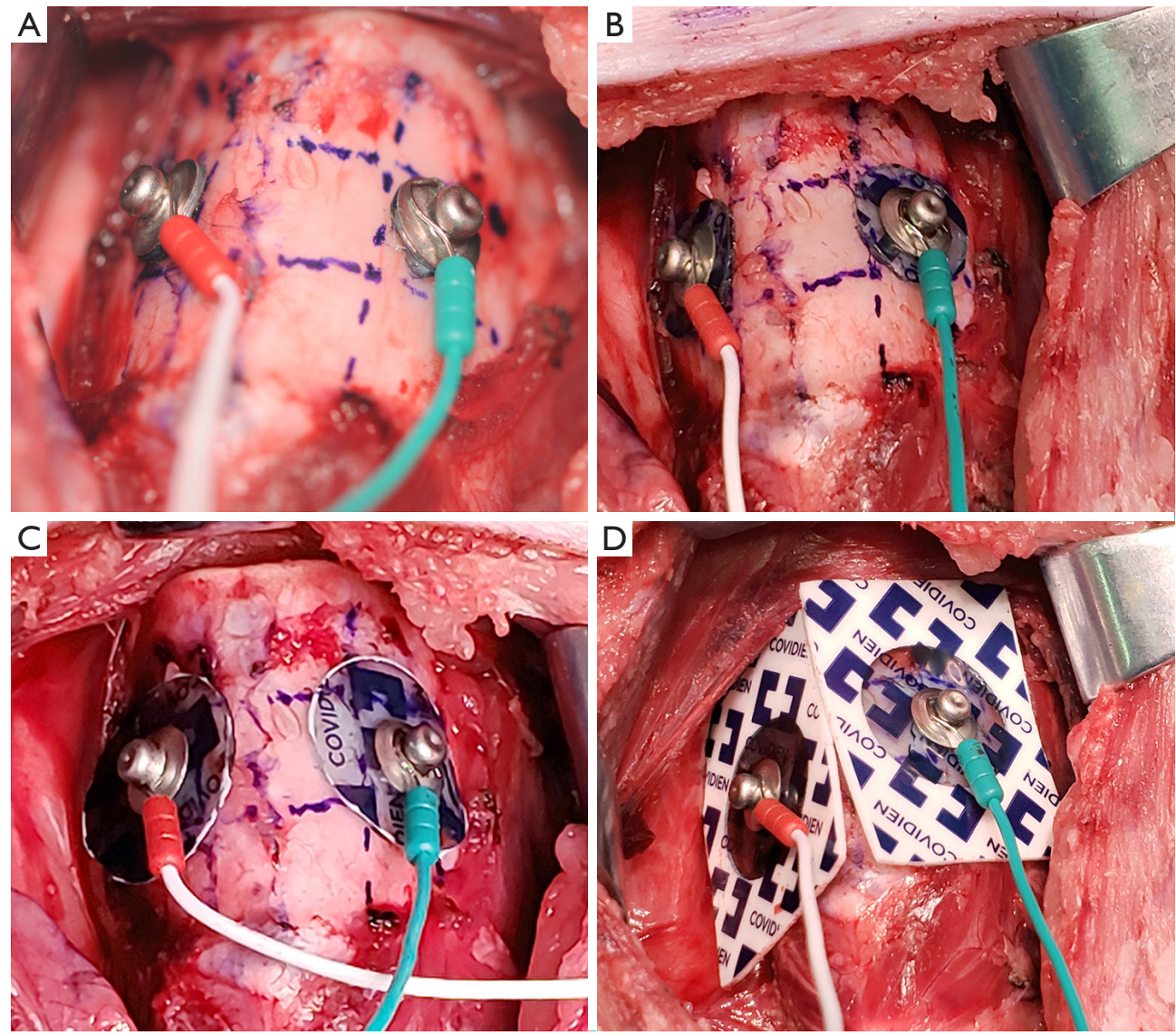

Figure 2 Different-sized ATCEs were centered on the surface of thyroid cartilage area No. 6. (A) $8 \mathrm{~mm}$; (B) $13 \mathrm{~mm}$; (C) $18 \mathrm{~mm}$; (D) total size (i.e., uncut). ATCEs, adhesive thyroid cartilage electrodes.

TC simultaneously. The channel leads' ET electrodes and ATCEs were connected to the monitoring system (16). The pair of ATCEs were single use. Thyroid cartilage needle recording electrodes and transcutaneous recording electrodes were used to compare the monitoring function with ATCEs $(13,15)$.

\section{Preparation of experiment and endpoints}

After surgical sterilization, an H-shaped incision in the middle of the neck was made to expose the thyroid gland, vagus nerve, RLN, and TC. The bilateral vagus nerve and RLNs were identified. C-IONM electrodes were carefully implanted bilaterally. The anterior TC tissues, including the sternothyroid and thyrohyoid muscles, were partially released and dissected. The cricothyroid and hypopharyngeal muscles that were attached to the surface of the thyroid cartilage were excised. The capillaries on the surface of cartilage perichondrium and the uneven cartilage perichondrium were removed. The surface of the TC was dried with gauze. In the pre-experiment, we determined that this treatment of the TC was advantageous for the placement of ATCE. Each side of the TC was divided into 9 areas with 2 transverse and 2 sagittal lines, as previously described (Figure 1) (13). The nerves were stimulated at the following 4 locations to record the latency profile (Figure 1): the vagus nerve just distal to the C-IONM electrode position, the most proximal exposed segment of the RLN, the middle portion of the RLN, and the RLN at the laryngeal point of entry. ATCEs were placed in the different TC areas and the 4 sites were stimulated simultaneously. The stability of the ATCE signal when manipulating the trachea and the ET was recorded. The following endpoints were assessed.

\section{ATCE adhesion characterization and test}

Because of anatomical difficulties of the TC, the areas 1, 
5 , and 7 to 9 were not selected for the placing and testing of all sizes of the ATCEs. Paired ATCEs were pasted on the TC in the number 2, 3, 4, and 6 areas (Figure 1). The EMG potentials from the ATCEs and ET electrodes were collected simultaneously. The optimal ATCE placement area was confirmed as the area with the highest EMG amplitude (Figure 1).

\section{ATCE geometrical analysis}

Four sizes of ATCEs were selected according to the outcome of the previous location testing (Figures 1,2). Calipers were used to measure the diameters of the ATCEs. They were 8, 13, and $18 \mathrm{~mm}$, and the entire (uncut) ECG adhesive electrodes (Figure 2). The EMG potentials from the ATCEs and ET electrodes were collected simultaneously. The optimal ATCE size was confirmed as the size associated with the highest recorded EMG amplitude.

\section{Mechanical tests: trachea and ET manipulation}

Six series of manipulations of the trachea and ET were applied to test the stability (anti-interference performance) of the ATCE signal, as follows (Figure 3): the trachea was pulled laterally, or the ET was pulled out superiorly $2 \mathrm{~cm}$ and then reinserted inferiorly $4 \mathrm{~cm}$; the ET was rotated contralaterally and then ipsilaterally relative to the stimulating C-IONM probe; and at last, the ET was adjusted to the initial position, as verified by video laryngoscope. The EMG potentials from the ATCEs and ET electrodes were registered simultaneously.

\section{Comparison of electrical properties monitoring function}

Four different EMG recording electrodes were used simultaneously for both intermittent and continuous monitoring; in addition to ATCEs and ET electrodes, $\mathrm{t}$ thyroid cartilage needle electrodes and transcutaneous recording electrodes were assessed $(13,18)$. RLN traction injury models were established for estimating their monitoring function (19). The RLN was dissected and a $1.5-\mathrm{mm}$ wide vascular rubber loop was positioned around the nerve and retracted slowly. EMG parameters were recorded by all four recording electrodes, with the ATCE in the optimal position. The traction was held until the alarm of the continuous IONM equipment sounded, at which point the traction was stopped, with standby until recovery of the electromyogram. Finally, traction of the RLN was implemented until loss of signal. Because the feasibility of nerve monitoring function of ET is acknowledged, so we compared the other electrodes with ET electrode for evaluation.

\section{Statistical analysis}

Four pigs were included in this study. We divided the bilateral thyroid cartilage of each pig into different areas. Each area provided 2 groups of data for each pig. Therefore, the ATCEs and ET electrodes could produce 8 groups of data from each area, respectively. Based on analysis using the SPSS version 22.0 software (IBM, Armonk, New York, USA), the data from each group exhibited normal distributions and homogeneity of variance. All data are expressed as mean \pm standard deviation. Statistical results were performed by one-way analysis of variance or Student's $t$ test, Kruskal-Wallis test was used between multiple independent samples using SPSS 22.0 software. The statistical significance was set at a $\mathrm{P}$ value $<0.05$.

\section{Data and code availability}

The data that support the findings of this study are available in the main text and the Information. The codes for EMG signal recording and analysis used in this study are available from the corresponding authors upon request.

\section{Results}

\section{ATCE location}

The ATCE amplitudes in stimulation areas number 2 and 3 were each significantly inferior to those of the ET electrodes; the ratios of ATCE to ET electrode amplitude values were 0.254 and 0.285 , respectively (Figure $4 A$, Table 1). In areas number 4 and 6, the ATCE and ET amplitudes were statistically similar: $1.002(\mathrm{P}=0.9917)$ and 1.003 ( $\mathrm{P}=0.987$ ), respectively (Figure 4A, Table 1 ). Area No. 6 was the optimal area to conduct the subsequent testing procedures, since it was at the TC center, distant from surrounding tissues, and easiest to place the electrodes.

\section{ATCE geometry}

There was no significant difference in amplitude profiles between the ET electrodes and ATCE by size $\left(\mathrm{P}_{0.8 \mathrm{~mm}}=0.721\right.$, $\left.\mathrm{P}_{1.3 \mathrm{~mm}}=0.387, \mathrm{P}_{1.8 \mathrm{~mm}}=0.583, \mathrm{P}_{\text {intact }}=0.915\right)$, and the 4 recordings 
A

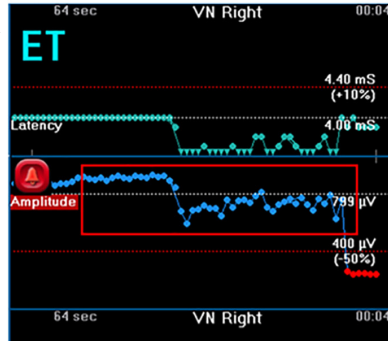

ATCE

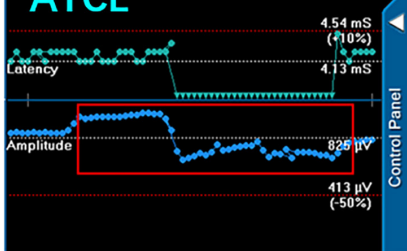

C
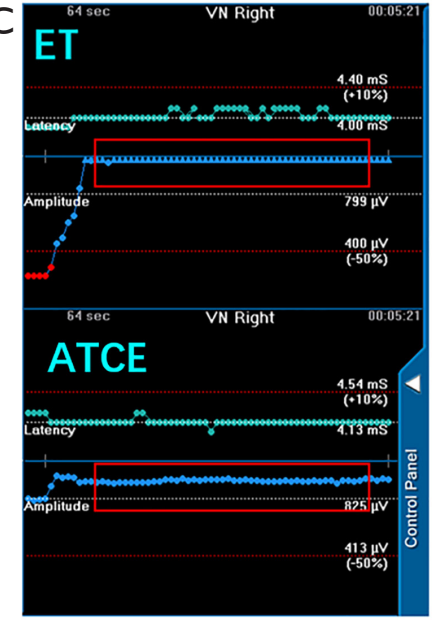

$\mathrm{E}$

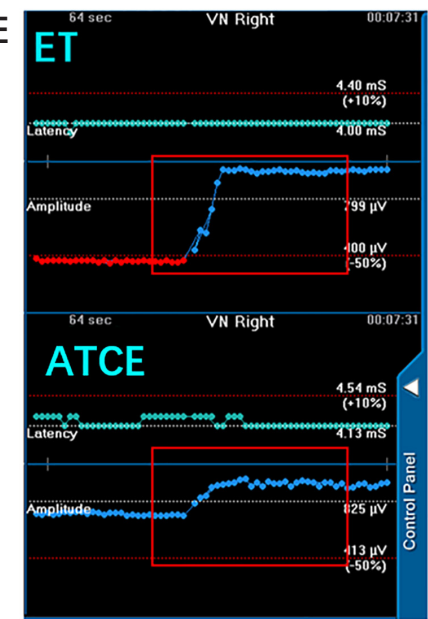

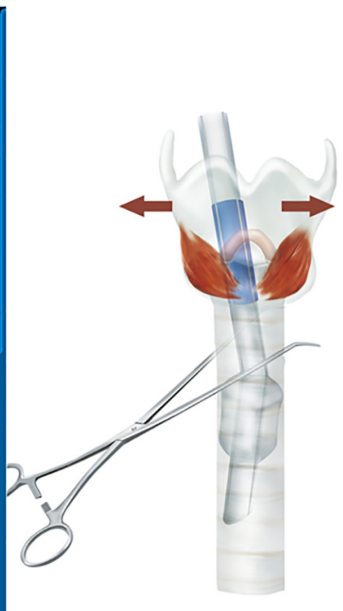
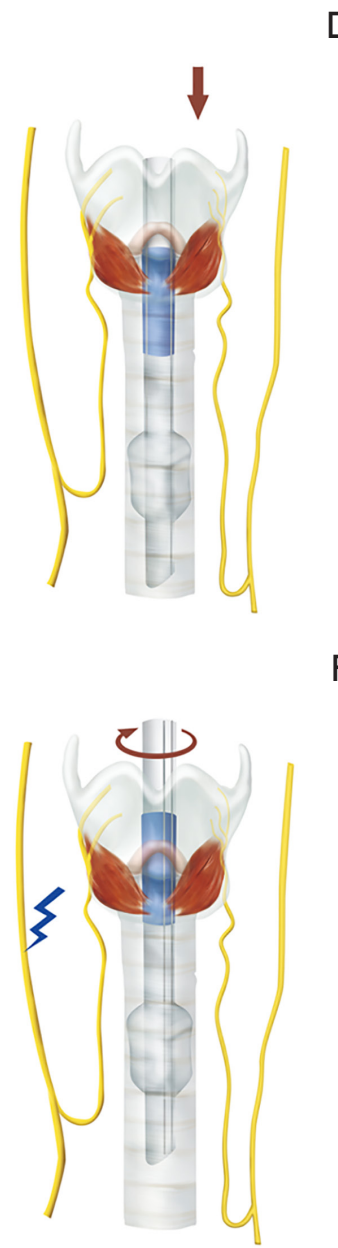
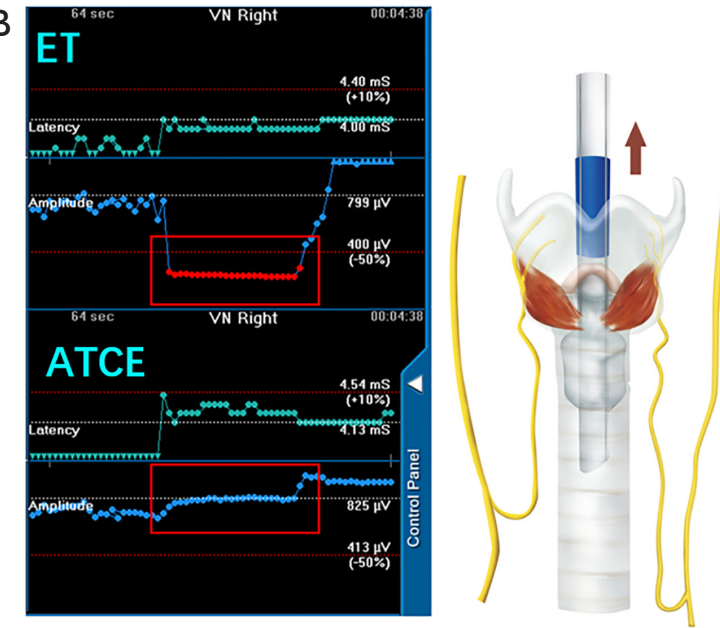

D
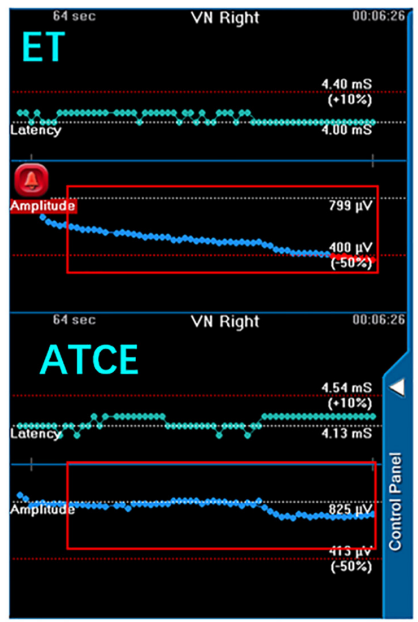

$\mathrm{F}$

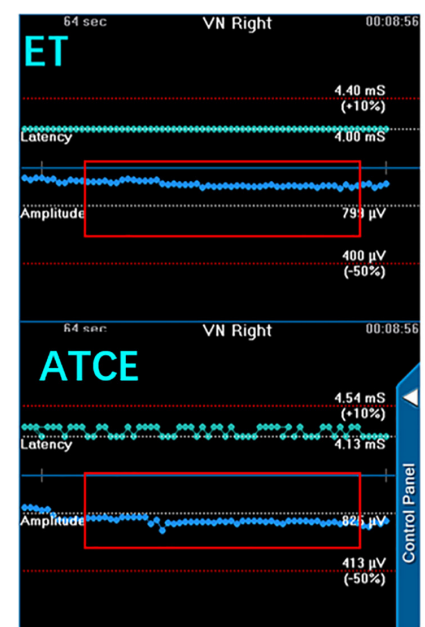

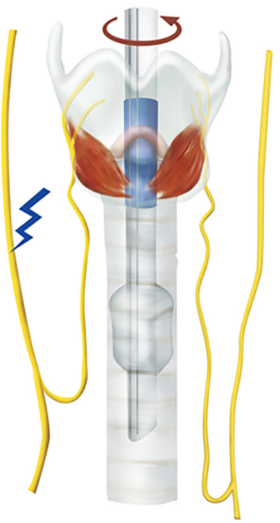

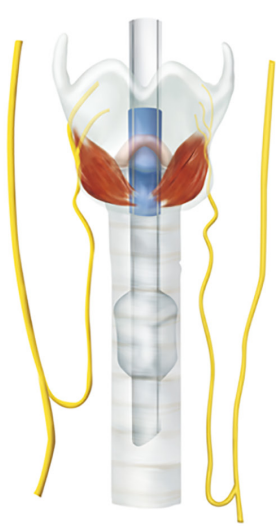

Figure 3 The EMG profile obtained from ATCEs and ET electrodes during the stability experiment. There were 6 manipulations in turn of the trachea and ET, as described below. (A) The trachea was pulled laterally, toward or away from the operator on each side with a forceps; (B) the ET was slowly displaced upward $2 \mathrm{~cm}$ and then (C) displaced inferiorly $4 \mathrm{~cm}$; (D) after the ET recovered to the initial position, the ET was rotated contralateral to the stimulating APS and subsequently (E) ipsilateral to the stimulating APS; (F) the ET was adjusted to the initial position with the video-laryngoscope. ATCEs, adhesive thyroid cartilage electrodes; APS, Automatic Periodic Stimulation; ET, endotracheal tube; EMG, electromyogram. 

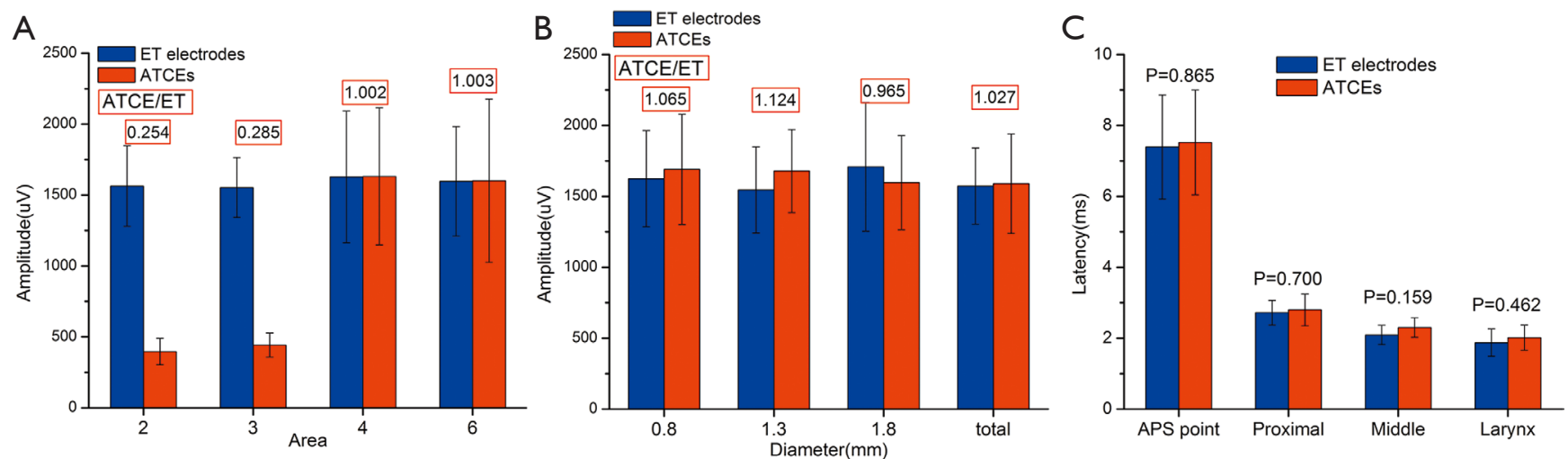

Figure 4 Graphic summaries of outcomes. (A) Correlation between EMG amplitudes and placement region of the ET electrodes and ATCEs; (B) association between EMG amplitudes and adhesive electrode sizes of ET electrodes and ATCEs; (C) latency recorded from ET electrodes and ATCEs on TC with different stimulating locations. ATCEs, adhesive thyroid cartilage electrodes; ET, endotracheal tube; EMG, electromyogram.

Table 1 The average amplitude and standard deviation (SD) for ATCEs and ET electrodes in all testing areas

\begin{tabular}{lccc}
\hline $\begin{array}{l}\text { Average } \\
\text { amplitude }(\mu \mathrm{V})\end{array}$ & ATCEs & ET electrodes & Ratio \\
\hline No. 2 & $396.750 \pm 93.411$ & $1,563.125 \pm 284.351$ & 0.254 \\
No. 3 & $443.125 \pm 85.242$ & $1,553.625 \pm 211.904$ & 0.285 \\
No. 4 & $1,631.250 \pm 483.157$ & $1,628.625 \pm 463.442$ & 1.002 \\
No. 6 & $1,600.625 \pm 575.635$ & $1,596.500 \pm 483.157$ & 1.003 \\
\hline
\end{tabular}

ATCEs, adhesive thyroid cartilage electrodes; ET, endotracheal tube.

Table 2 The average amplitude and standard deviation (SD) of the different electrodes used with the different sizes of ATCEs

\begin{tabular}{llll}
\hline $\begin{array}{l}\text { Diameter of } \\
\text { ATCEs }\end{array}$ & ATCEs $(\mu \mathrm{V})$ & ET electrodes $(\mu \mathrm{V})$ & Ratio \\
\hline $0.8 \mathrm{~mm}$ & $1,691.375 \pm 388.862$ & $1,625.000 \pm 339.240$ & 1.065 \\
$1.3 \mathrm{~mm}$ & $1,678.250 \pm 292.592$ & $1,545.250 \pm 303.332$ & 1.124 \\
$1.8 \mathrm{~mm}$ & $1,596.625 \pm 331.513$ & $1,708.375 \pm 453.699$ & 0.965 \\
Intact & $1,590.000 \pm 350.794$ & $1,573.000 \pm 268.449$ & 1.027 \\
\hline $\begin{array}{l}\text { ATCEs, adhesive thyroid cartilage electrodes; ET, endotracheal } \\
\text { tube. }\end{array}$ & & & \\
\end{tabular}

were comparable ( $\mathrm{P}=0.882$; Figure $4 B$, Table 2$)$. The $13-\mathrm{mm}$ ATCE had proper area contact and was more firmly located with less intraexperimental hindrance, and thus was the optimal geometry and chosen for subsequent testing.
Table 3 The average latency and standard deviation (SD) received by different electrodes at nerve stimulation points

\begin{tabular}{llll}
\hline $\begin{array}{l}\text { Nerve stimulation } \\
\text { points }\end{array}$ & ATCEs $(\mu \mathrm{V})$ & ET electrodes $(\mu \mathrm{V})$ & P value \\
\hline APS point & $7.523 \pm 1.476$ & $7.395 \pm 1.471$ & 0.865 \\
Proximal & $2.799 \pm 0.449$ & $2.720 \pm 0.346$ & 0.700 \\
Middle & $2.299 \pm 0.274$ & $2.095 \pm 0.273$ & 0.159 \\
Larynx & $2.016 \pm 0.356$ & $1.876 \pm 0.384$ & 0.462 \\
\hline
\end{tabular}

ATCEs, adhesive thyroid cartilage electrodes; ET, endotracheal tube; APS, Automatic Periodic Stimulation.

\section{Conductivity}

There was no significant difference in latencies between the ATCE and ET electrodes at each of the 4 nerve stimulation points $(\mathrm{P}=0.865,0.700,0.159$, and 0.462 , respectively; Figure 4C, Table 3).

\section{Adhesiveness forces: tracheal and ET manipulation}

Manipulation of the trachea was associated with unstable EMG amplitudes from the ET electrodes, but the EMG amplitudes from the ATCEs were very stable (Figure $3 A$ ). With displacements or rotations of the ET, the EMG amplitudes from the ET electrodes were affected more significantly compared with the ATCE (Figure 3B, C,D,E,F). The ATCE signals were stable during the entire series of surgery manipulations (Figure 3). 

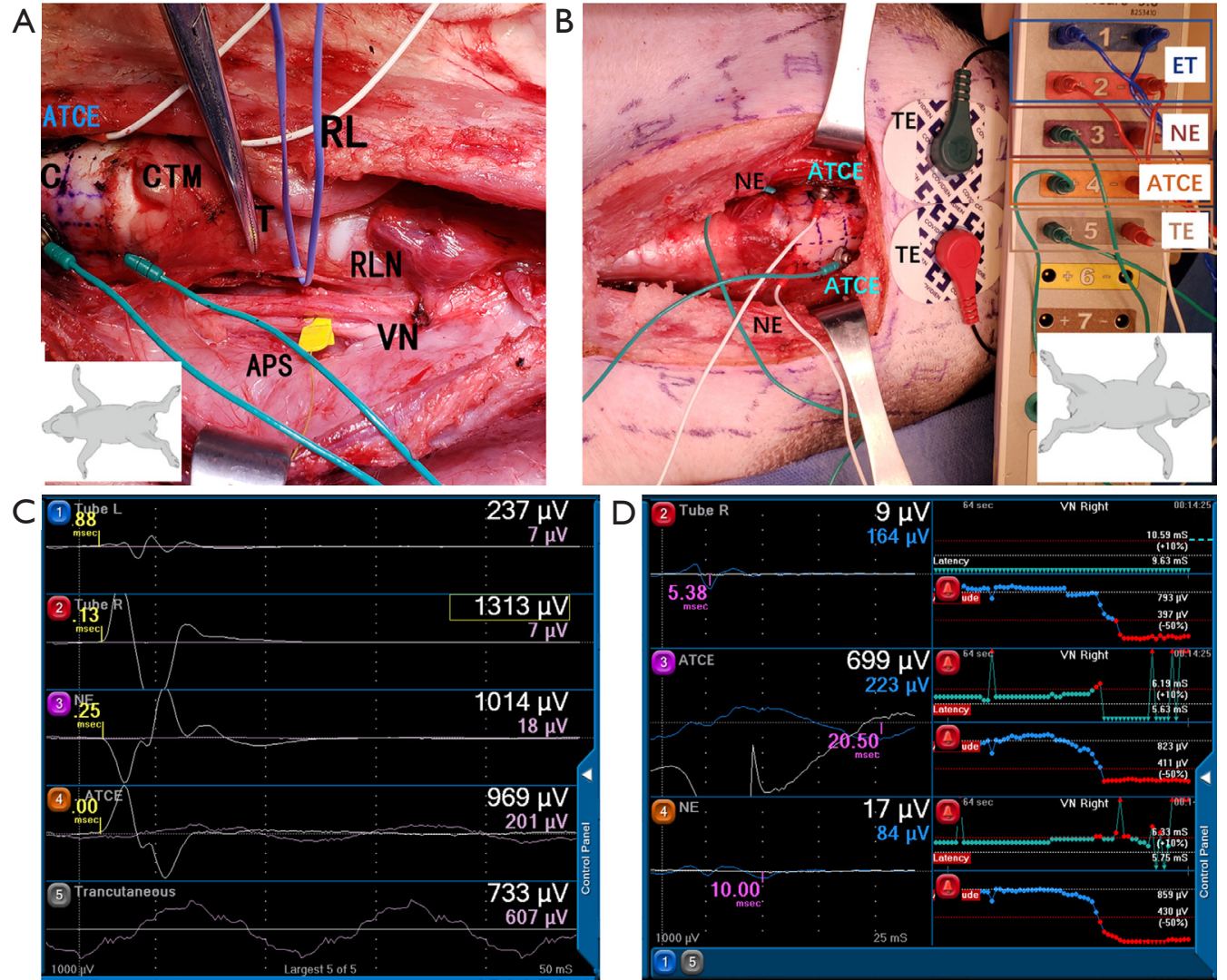

Figure 5 Recurrent laryngeal nerve traction model with four different recording electrodes and their performances on monitoring. (A) A recurrent laryngeal nerve traction injury model; (B) simultaneous neural monitoring by ATCEs, transcutaneous recording electrodes, needle electrodes, and ET electrodes; (C) intermittent monitoring with 4 different recording electrodes; (D) continuous monitoring with ATCE, needle, and ET electrodes during nerve pulling. ATCEs, adhesive thyroid cartilage electrodes; CTM, cricothyroid muscle; RL, rubber loop; NE, needle electrode; T, trachea; TE, transcutaneous recording electrodes; ET, endotracheal tube.

\section{Biopotential detection comparison}

When all 4 recording electrodes were activated simultaneously, ATCEs, ET electrodes, and TC needle electrodes could be used normally during both intermittent and continuous monitoring, but the transcutaneous recording electrodes could not obtain effective EMG signals. In the test of the RLN traction injury model, when the RLNs were retracted, all the ATCEs, ET electrodes and TC needle electrodes could detect the change of EMG profiles early with C-IONM (Figure 5).

\section{Long-term monitoring}

The ATCE electrodes were used for long-term monitoring, supported by the high-quality EMG signals after continuous use for 5 hours on average of each porcine model.

\section{Discussion}

Using ATCEs or so-called anterior laryngeal electrodes for monitoring the RLN has been previously reported in an animal porcine model (22) and also a clinical study (23). This study further compared the EMG signal stability recorded by different sizes of ATCEs and different TC locations for determining the optimal placement of ATCEs when monitoring the RLN. The EMG amplitudes and latencies detected by ATCEs and ET electrodes were compared, using both intermittent and continuous monitoring. Checking the whole exposed RLN and vagus nerve with prospective comparison of 2 techniques was another original feature of this study.

According to the classification of RLN injury mechanism, RLN palsy can be divided into sever injury and non-sever injury, and non-sever injury is more common 
during surgery. RLN palsy caused by non-severed injury can be divided into visible injury, such as thermal injury, ligation injury, and non-visible injury, such as traction injury. The latter is the main type of our study, because this kind of injury is the most common type of RLN palsy in surgery, and it is also the only one that can be predicted by IONM and it can help the recovery of nerve function.

Through IONM, we can avoid the occurrence of this kind of injury as far as possible. The different recording electrodes involved in this study will affect the monitoring function of the nerve monitoring instrument in different degrees, so that the ability of IONM to prevent nerve injury is different.

This work comparing responses from common endotracheal tube (vocal fold surface) electrodes and transcutaneous recording electrodes versus thyroid-laminaplaced electrodes has the potential to add novel information useful towards expanding the electrode options for IONM. For the first time, 4 different recording electrodes, including ATCEs, ET electrodes, TC needle electrodes, and transcutaneous recording electrodes, were used to test the RLN monitoring function simultaneously.

This newly designed recording electrode had demonstrably excellent monitoring function during thyroid surgery. It has the potential to replace the classic ET-embed superficial electrodes during thyroid surgery, as it is cheap, stable among the changes of EMG signal during surgical manipulations (15), and has demonstrated particularly good anti-interference ability. Surgical manipulations did not affect the EMG, as is characteristic of ET electrodes (10).

In order to better simulate the process of IONM, in this study, we strictly according to the four-step intraoperative standard procedure. First step: V1 signal, before exposure of RLN, the vagus nerve was stimulated at the lower thyroid pole level to obtain EMG. Second step: R1 signal, before dissecting the RLN, stimulating the exposed segment to obtain EMG. Third step: R2 signal, after the RLN was dissected, the EMG signal was obtained by stimulating the proximal end of the exposed segment. Fourth step: V2 signal, after complete hemostasis, the vagus nerve was stimulated again to obtain EMG signal.

During monitored thyroid surgery, stable and welldelineated EMG profiles are critical. The EMG amplitudes are the most sensitive index, as they represent nerve function in real time and help prevent laryngeal nerve injury. However, a malpositioned trachea tube can lead to poor contact of the electrodes with the vocal cords, the results of which can misguide surgeons. For example, the ET can be displaced after the patient has been fully positioned with head extension, or during the surgery because of in-field traction on the trachea $(8,9,24)$. This seriously affects the application of IONM. The clinical application value of real-time monitoring of RLN in highrisk and complex thyroid surgery is that, the RLNs in these cases are more vulnerable, real-time monitoring can show the nerve function through amplitude and other EMG parameters without delay, so that the surgeons can find any little damage and take reasonable action in time. Thereby, it can reduce the rate of nerve injury better.

During the test of ATCE stability, we consulted the study of Kim et al. (10), in which tube rotation and insertion malpositions were replicated during IONM. Their study showed that any malposition of the ET can affect the position between the ET electrodes and vocal cords, leading to monitoring failure during surgery. Their study tested the common surgical manipulations that can occur during monitored thyroid surgery, and simulations of poor EMG profiles were produced.

In the present study, with every tested manipulation of the trachea or ET, the EMG profile recorded by the ATCEs showed greater signal stability than did the ET electrodes. When the ET was readjusted to the initial position using the video laryngoscope, the EMG profiles from both the ET electrodes and the ATCEs were similar to the baseline profiles. This indicated that the EMG profile from the ATCEs remained stable and resisted surgical interference.

Wu et al. (15) recently evaluated transcutaneous adhesive pregelled surface electrodes for recording evoked EMG signals relative to surface electrodes on the ET. In both groups, typically evoked laryngeal EMG waveforms were recorded from the RLNs under stimulation with $1 \mathrm{~mA}$. However, under tracheal displacement, the EMG signals of the experimental group remained stable, while those of the control varied significantly. The study confirmed the feasibility of the transcutaneous approach, but the authors recognized the need for new electrode designs to improve EMG amplitudes, and the identification of optimal electrode positions (15). We recommend placing the ATCEs in the TC area number 6, because here the ATCE resulted in an EMG amplitude that was equivalent to that of the ET electrodes, and the initial EMG amplitudes were high enough for monitored thyroid surgery. High amplitudes of $\mathrm{V} 1$ and $\mathrm{R} 1$, are suggested in the IONM guidelines $(>500 \mu \mathrm{V})(6,10,25)$. With the newly designed recording electrode used in the present study, even the EMG baseline for continuous IONM can be easily established. 
Furthermore, stable EMG profiles are important for nerve monitoring (7-10,13,24). Kim et al. (10) reported the effect of positional changes on EMG potentials during IONM with ET electrodes. ATCEs were not affected by tracheal manipulation. Similarly to TC needle electrodes, ATCEs are firmly affixed to the surface of the TC and within the surgical field. Thus, displacement can be easily observed $(6,11,15,19,26-28)$. Latency is an independent variable that is associated with ET electrode position. During the test describe here, the latencies of all the stimulation points on the nerve were comparable to the results from ET electrodes. Latencies did not differ between ATCE and ET recording approaches across all of the nerve stimulation locations. This indicated again that the ATCEs have the same monitoring function as conventional ET electrodes.

Furthermore, TC area number 6 was easily located, dissected, assembled, and inspected during surgery. This was confirmed by a previous TC specimen analysis in an anatomical study (13). Area number 6 is on the center surface of the TC, and additional dissection of this area is hardly required. The vocal cord is proximally located in half of the TC and thyroarytenoid muscles and lateral cricoarytenoid muscles. These muscles are innervated by the RLN. In areas number 4 and 6, the ATCE has a superior ability to register larger ranges of muscular activity as compared with other areas on the TC. The other TC placement areas have intrinsic limitations for ATCE placement.

In this study, the size of the ATCE had no significant effect on the amplitude or latency. In fact, the 13-mm ATCE had a proper area contact, the firmest hold, and the least hindrance in the surgical field. Latencies did not change with the diameter of the ATCE or placement area $(6,13,15,26)$.

Although the optimal placement location and electrode size were recommended according to the results from this experiment, it should be noted that areas 1,5 , and 7 to 9 proved difficult for stably fixing the electrode in place in this study. Thus, no testing was carried out in those areas. The test results for areas 2 and 3 showed poor monitoring capabilities, and the EMG signal that was obtained was lower than that for areas 4 and 6 . Therefore, there was no application advantage for areas 2 and 3. Although it can be observed from the results that the ability to receive EMG signals in areas 4 and 6 were quite similar, the location of area 4 was lower and closer to the thyroid surgical area. Thus, more dissection steps might be needed during the surgery, as interference with the surgical procedures on the thyroid were more likely to occur. Concerning the different electrode sizes, we found that there were no statistical differences among the EMG recording abilities for the different sizes of ATCEs or ET electrodes. Therefore, we focused our attention to the convenience and stability of the different sizes of ATCEs. In this experiment, we observed that the larger the ATCE size was, the larger the contact area with the thyroid cartilage and the stronger the fixation. However, it was easier to cover more non-cartilage tissue, and the variability in the non-cartilage tissue surface could affect the stability of the ATCE placement. Finally, we chose a 13-mm diameter ATCE, which was slightly smaller than the area of the thyroid cartilage on one side. We believe that in the practical application, a product similar to that used in this experiment could be made according to the size of the human thyroid cartilage.

Compared with TC needle electrodes or surrounding muscle needle electrodes, ATCEs are noninvasive. Although the skin-surface electrodes modified for testing in this application may be appropriately described as noninvasive for their intended use, it is inappropriate to claim that electrode placement for IONM on the thyroid lamina is truly noninvasive (even in the context of an open neck). The authors acknowledge that extra dissection is required for electrode placement (e.g., removal of some or all of the thyrohyoid muscle, perichondrium, vasculature, etc.). While these electrodes may be less invasive than needle electrodes passed through the cartilage, this does not justify classifying them as noninvasive.

ATCEs appear be more convenient to set up and use $(7,29)$. In addition, ATCEs can be more cost-effective for production $(16,17,30-31)$. The expense of the ET has limited the application of IONM in some regions $(16,17)$. In China, the overall cost of an ET, stimulating probe, APS electrodes, and ground and return needle electrodes is prohibitively expensive, and using hand-trimmed ECG electrodes might produce substantial savings.

No model perfectly resembles the human neck and larynx, and this should be considered when working with porcine models. The larynx of a pig closely resembles that of a human and has been used as the classic model of intraoperative RLN monitoring with in many influential studies $(10,13-15,19,20,22,26)$. Also, anatomists have used the porcine larynx to study human laryngeal structure. Jiang et al. (32) observed that in the thickness and structure of the vocal fold, the porcine larynx most closely resembles that of a human as compared to other common animal models. In 
addition, there is enough contact area in the porcine thyroid cartilage, which is an advantage for TC recording electrode study. Finally, the study lasted for more than 5 hours. Therefore, ATCE can be used for long-term monitoring.

Some limitations of this study should also be mentioned. First, this study used a small sample size, and a larger sample of animals may provide greater statistical power. Furthermore, adhesive surface electrodes may be limited by their imperfect compliance with the size or shape of the TC, especially during surgeon movements, dissection, and secretions, resulting in high interfacial impedance and motion artifacts. In this study, an ECG adhesive electrode was used to affix onto the TC, but a specific conductive material with more biocompatibility that can adhere to the surface or TC is still required. Use of a transcartilage electrode in humans needs to be investigated with regard to morphological variations associated with gender and age that may affect its utility. The limitations of surface electrodes also include displacement and exposure issues (i.e., the need to expose the entire thyroid cartilage which, particularly for minimally invasive approaches, is rarely done), firm stability during clinical case, possible predisposition to bleeding, and muscle damage upon removal. In addition, the electrodes are prone to displacement in cases where a new facet is added to any "loss of signal" troubleshooting algorithm. Biocompatibility testing was not evaluated in the present work, and further TC mucosa irritation assessment after attaching the ATCE to the mucosa of the experimental animals is required. Moreover, we did not stimulate the external branch of the superior laryngeal nerve (EBSLN). Results regarding EBSLN monitoring may be related to those of RLN monitoring. There is also a lack of data regarding combined EMG events. Finally, there are several descriptions for latency in the literature, and we adopted only one in the evaluation of the present experimental study $(6,25)$.

\section{Conclusions}

Nowadays, the limitations of IONM are mainly in three aspects, they are simplification of system, optimization of equipment and standardization of application methods. To address the above limitations, researchers and clinicians have conducted a large number of clinical and animal experiments. This study aims to design and apply a recording electrode which is easier to install, less damage to tissue, cheaper, and more widely used, so as to avoid the limitations of traditional ET and needle electrode.

This preliminary study used a porcine model to evaluate the application of ATCEs affixed onto the TC, comparing the ability of transcutaneous recording, needle, and ET electrodes to generate an EMG profile of the RLN. The EMG amplitudes and latencies detected by ATCEs and ET electrodes were compared with both intermittent and continuous monitoring. According to the results, the feasibility and stability of ATCEs were confirmed, and amplitudes and latencies acquired by the ATCEs and ET electrodes were consistent. The EMG profiles from the ATCEs were more stable during manipulations to the trachea and ET compared with those of the ET electrodes. The current results suggest that the ATCE may be an evaluation of choice for RLN function in thyroid surgery.

\section{Acknowledgments}

Funding: None.

\section{Footnote}

Reporting Checklist: The authors have completed the ARRIVE reporting checklist. Available at http://dx.doi. org/10.21037/atm-21-1229

Data Sharing Statement: Available at http://dx.doi. org/10.21037/atm-21-1229

Conflicts of Interest: All authors have completed the ICMJE uniform disclosure form (available at http://dx.doi. org/10.21037/atm-21-1229). The authors have no conflicts of interest to declare.

Ethical Statement: The authors are accountable for all aspects of the work in ensuring that questions related to the accuracy or integrity of any part of the work are appropriately investigated and resolved. Experiments were performed under a project license (No.: 2019072801) granted by ethics board of Jilin Provincial Key Laboratory of Surgical Translational Medicine, in compliance with national or institutional guidelines for the care and use of animals.

Open Access Statement: This is an Open Access article distributed in accordance with the Creative Commons Attribution-NonCommercial-NoDerivs 4.0 International License (CC BY-NC-ND 4.0), which permits the noncommercial replication and distribution of the article with the strict proviso that no changes or edits are made and the 
original work is properly cited (including links to both the formal publication through the relevant DOI and the license). See: https://creativecommons.org/licenses/by-nc-nd/4.0/.

\section{References}

1. Jeannon JP, Orabi AA, Bruch GA, et al. Diagnosis of recurrent laryngeal nerve palsy after thyroidectomy: a systematic review. Int J Clin Pract 2009;63:624-9.

2. Haugen BR, Alexander EK, Bible KC, et al. 2015 American Thyroid Association Management Guidelines for Adult Patients with Thyroid Nodules and Differentiated Thyroid Cancer: The American Thyroid Association Guidelines Task Force on Thyroid Nodules and Differentiated Thyroid Cancer. Thyroid 2016;26:1-133.

3. Terris DJ, Snyder S, Carneiro-Pla D, et al. 2013 American Thyroid Association Statement on Outpatient Thyroidectomy. Thyroid 2013;23:1193-202.

4. Chen AY, Bernet VJ, Carty SE, et al. 2014 American thyroid association statement on optimal surgical management of goiter. Thyroid 2014;24:181-9.

5. Shindo ML, Caruana S, Kandil E, et al. 2014 Management of invasive well-differentiated thyroid cancer an American Head and Neck society consensus statement. Head Neck 2014;36:1379-90.

6. Randolph GW, Dralle H; International Intraoperative Monitoring Study Group, et al. Electrophysiologic recurrent laryngeal nerve monitoring during thyroid and parathyroid surgery: international standards guideline statement. Laryngoscope 2011;121 Suppl 1:S1-16.

7. Chiang FY, Lu IC, Chang PY, et al. Comparison of EMG signals recorded by surface electrodes on endotracheal tube and thyroid cartilage during monitored thyroidectomy. Kaohsiung J Med Sci 2017;33:503-9.

8. Tsai CJ, Tseng KY, Wang FY, et al. Electromyographic endotracheal tube placement during thyroid surgery in neuromonitoring of recurrent laryngeal nerve. Kaohsiung J Med Sci 2011;27:96-101.

9. Lu IC, Chu KS, Tsai CJ, et al. Optimal depth of NIM EMG endotracheal tube for intraoperative neuromonitoring of the recurrent laryngeal nerve during thyroidectomy. World J Surg 2008;32:1935-9.

10. Kim HY, Tufano RP, Randolph G, et al. Impact of positional changes in neural monitoring endotracheal tube on amplitude and latency of electromyographic response in monitored thyroid surgery: results from the porcine experiment. Head Neck 2016;38 Suppl 1:E1004-8.

11. Wu CW, Wang MH, Chen CC, et al. Loss of signal in recurrent nerve neuromonitoring: causes and management. Gland Surg 2015;4:19-26.

12. Schneider R, Machens A, Sekulla C, et al. Twenty-year experience of paediatric thyroid surgery using intraoperative nerve monitoring. Br J Surg 2018;105:996-1005.

13. Zhao Y, Li C, Zhang D, et al. Experimental study of needle recording electrodes placed on the thyroid cartilage for neuromonitoring during thyroid surgery. Br J Surg 2019;106:245-54.

14. Van Slycke S, Van Den Heede K, Magamadov K, et al. New placement of acquisition electrodes on the thyroid cartilage in intra-operative neuromonitoring during thyroid surgery. Krakow, Poland: The First World Congress of Neural Monitoring in Thyroid and Parathyroid Surgery; 2015.

15. Wu CW, Chiang FY, Randolph GW, et al. Transcutaneous recording during intraoperative neuromonitoring in thyroid surgery. Thyroid 2018;28:1500-7.

16. Dionigi G, Bacuzzi A, Boni L, et al. Visualization compared with neuromonitoring of recurrent laryngeal nerves during thyroidectomy: what about the costs? World J Surg 2012;36:748-54.

17. Kim HY, Chai YJ, Freni F, et al. Cost-Effectiveness Estimate for Neural Monitoring in Thyroid Surgery. J Endocr Surg 2018;18:10-20.

18. Kilkenny C, Browne WJ, Cuthill IC, et al. Improving Bioscience Research Reporting: The ARRIVE Guidelines for Reporting Animal Research. PLoS Biol 2010;8:e1000412.

19. Wu CW, Dionigi G, Sun H, et al. Intraoperative neuromonitoring for the early detection and prevention of RLN traction injury in thyroid surgery: a porcine model. Surgery 2014;155:329-39.

20. Lin YC, Dionigi G, Randolph GW, et al. Electrophysiologic monitoring correlates of recurrent laryngeal nerve heat thermal injury in a porcine model. Laryngoscope 2015;125:E283-90.

21. Janssen PF, Brölmann HA, Huirne JA. Effectiveness of electrothermal bipolar vessel-sealing devices versus other electrothermal and ultrasonic devices for abdominal surgical hemostasis: a systematic review. Surg Endosc 2012;26:2892-901.

22. Wu CW, Chiang FY, Randolph GW, et al. Feasibility of Intraoperative Neuromonitoring During Thyroid Surgery Using Transcartilage Surface Recording Electrodes. Thyroid 2018;28:1508-16.

23. Liddy W, Lawson BR, Barber SR, Kamani D, Shama M, Soylu S, Wu CW, Chiang FY, Scharpf J, Barczynski M, 
Dralle H, et al. Anterior laryngeal electrodes for recurrent laryngeal nerve monitoring during thyroid and parathyroid surgery: New expanded options for neural monitoring. Laryngoscope 2018;128:2910-5.

24. Bacuzzi A, Dionigi G, Del Bosco A, et al. Anaesthesia for thyroid surgery: perioperative management. Int J Surg 2008;6 Suppl 1:S82-5.

25. Lorenz K, Sekulla C, Schelle J, et al. What are normal quantitative parameters of intraoperative neuromonitoring (IONM) in thyroid surgery? Langenbecks Arch Surg 2010;395:901-9.

26. Brauckhoff $\mathrm{K}$, Aas T, Biermann M, et al. EMG changes during continuous intraoperative neuromonitoring with sustained recurrent laryngeal nerve traction in a porcine model. Langenbecks Arch Surg 2017;402:675-81.

27. Brauckhoff K, Vik R, Sandvik L, et al. Impact of EMG Changes in Continuous Vagal Nerve Monitoring in High-Risk Endocrine Neck Surgery. World J Surg 2016;40:672-80.

28. Schneider R, Randolph GW, Barczynski M, et al.

Cite this article as: Zhao Y, Zhang D, Zhou L, Li S, Wang T, Li F, Han Y, Dionigi G, Sun H. Proprieties of adhesive surface arrays to thyroid cartilage for recurrent laryngeal nerve monitoring. Ann Transl Med 2021;9(8):690. doi: 10.21037/atm$21-1229$
Continuous intraoperative neural monitoring of the recurrent nerves in thyroid surgery: a quantum leap in technology. Gland Surg 2016;5:607-16.

29. Meyer T, Hamelmann W, Timmermann W, et al. The Advantages and Disadvantages of Nerve Monitoring During Thyroid Surgery in Childhood. Eur J Pediatr Surg 2006;16:392-5.

30. Horne SK, Gal TJ, Brennan JA. Prevalence and patterns of intraoperative nerve monitoring for thyroidectomy. Otolaryngol Head Neck Surg 2007;136:952-6.

31. Sturgeon C, Sturgeon T, Angelos P. Neuromonitoring in thyroid surgery: attitudes, usage patterns, and predictors of use among endocrine surgeons. World J Surg 2009;33:417-25.

32. Jiang JJ, Raviv JR, Hanson DG. Comparison of the phonation-related structures among pig, dog, white-tailed deer, and human larynges. Ann Otol Rhinol Laryngol 2001;110:1120-5.

(English Language Editor: J. Gray) 